\title{
Evidence of one-dimensional magnetic heat transport in the triangular-lattice antiferromagnet $\mathrm{Cs}_{2} \mathrm{CuCl}_{4}$
}

\author{
E. Schulze,${ }^{1,2}$ S. Arsenijevic, ${ }^{1}$ L. Opherden, ${ }^{1,2}$ A. N. Ponomaryov $\odot,{ }^{1}$ J. Wosnitza, ${ }^{1,2}$ \\ T. Ono, ${ }^{3} \mathrm{H}$. Tanaka, ${ }^{4}$ and S. A. Zvyagin $\oplus^{1, *}$ \\ ${ }^{1}$ Dresden High Magnetic Field Laboratory (HLD-EMFL) and Würzburg-Dresden Cluster of Excellence ct.qmat, \\ Helmholtz-Zentrum Dresden-Rossendorf, 01328 Dresden, Germany \\ ${ }^{2}$ Institute for Solid State and Materials Physics, TU Dresden, 01062 Dresden, Germany \\ ${ }^{3}$ Department of Physical Science, Osaka Prefecture University, Osaka 599-8531, Japan \\ ${ }^{4}$ Department of Physics, Tokyo Institute of Technology, Tokyo 152-8551, Japan
}

(Received 12 August 2019; published 20 November 2019)

\begin{abstract}
We report on low-temperature heat-transport properties of the spin-1/2 triangular-lattice antiferromagnet $\mathrm{Cs}_{2} \mathrm{CuCl}_{4}$. Broad maxima in the thermal conductivity along the three principal axes, observed at about $5 \mathrm{~K}$, are interpreted in terms of the Debye model, including the phonon umklapp scattering. For thermal transport along the $b$ axis, we found a pronounced field-dependent anomaly, close to the transition into the three-dimensional long-range-ordered state. No such anomalies were found for the transport along the $a$ and $c$ directions. We argue that this anisotropic behavior is related to an additional heat-transport channel through magnetic excitations, that can best propagate along the direction of the largest exchange interaction. In addition, peculiarities of the heat transport of $\mathrm{Cs}_{2} \mathrm{CuCl}_{4}$ in magnetic fields up to the saturation field and above are discussed.
\end{abstract}

DOI: 10.1103/PhysRevResearch.1.032022

Frustrated magnets are known to be an excellent playground to test fundamental concepts of condensed matter physics and quantum mechanics [1-6]. Spin-1/2 Heisenberg antiferromagnets (AFs) on triangular lattices have attracted particular attention, representing an important class of lowdimensional (low-D) frustrated magnets and allowing one to probe effects of the geometrical frustration, magnetic order, and quantum fluctuations in strongly correlated spin systems. Particularly, this interest was stimulated by the idea of the "resonating valence bond" (RVB) ground state for an AF system of spins on a triangular layer lattice [7]. This quantum-disordered ground state was proposed to be a $2 \mathrm{D}$ fluid of resonating spin-singlet pairs, with the elementary excitation spectrum formed by fractionalized mobile quasiparticles, spinons. Since that time, searching for experimental realizations of the $2 \mathrm{D}$ quantum spin liquid appears to be one of the central topics in quantum physics.

Among others, the triangular-lattice $\mathrm{AF} \mathrm{Cs}_{2} \mathrm{CuCl}_{4}$ has drawn a particularly great deal of attention. Magnetic $\mathrm{Cu}^{2+}$ ions in $\mathrm{Cs}_{2} \mathrm{CuCl}_{4}$ form a quasi-2D lattice with the exchange coupling parameters $J$ along the $b$ axis (regarded as the chain direction) and $J^{\prime}$ along the zigzag bonds in the $b c$ plane (Fig. 1). Its spin Hamiltonian reads

$$
\mathcal{H}_{2 \mathrm{~d}}=\sum_{i} J \vec{s}_{i} \cdot \vec{s}_{i+1}+\sum_{k} J^{\prime} \vec{s}_{k} \cdot \vec{s}_{k+1}+\mathcal{H}_{\delta},
$$

\footnotetext{
*Corresponding author: s.zvyagin@hzdr.de
}

Published by the American Physical Society under the terms of the Creative Commons Attribution 4.0 International license. Further distribution of this work must maintain attribution to the author(s) and the published article's title, journal citation, and DOI. where $\vec{s}_{i}$ are the spins along and $\vec{s}_{k}$ between the chains (along the zigzag bonds), and $\mathcal{H}_{\delta}$ represents various possible, usually small, contributions [such as interlayer and the Dzyaloshinskii-Moriya (DM) interactions]. High-field electron spin resonance (ESR) measurements [8] revealed $J / k_{B}=$ $4.7 \mathrm{~K}$ and $J^{\prime} / k_{B}=1.4 \mathrm{~K}\left(k_{B}\right.$ is the Boltzmann constant), yielding the ratio $J^{\prime} / J \approx 0.3$, which is in good agreement with parameters estimated from neutron-scattering experiments [9]. The interlayer coupling in $\mathrm{Cs}_{2} \mathrm{CuCl}_{4}$ is smaller than $J$ and $J^{\prime}$ by more than one order of magnitude, $J^{\prime \prime}=0.13 \mathrm{~K}$ [9]. At $T_{N}=0.62 \mathrm{~K}, \mathrm{Cs}_{2} \mathrm{CuCl}_{4}$ undergoes a phase transition into a cycloidal long-range-ordered state with an incommensurate wave vector $q=(0,0.472,0)$ [10]. Saturation fields of $\mathrm{Cs}_{2} \mathrm{CuCl}_{4}\left(\mu_{0} H_{\mathrm{sat}}=8.44,8.89\right.$, and $8 \mathrm{~T}$, along the $a, b$, and $c$ axes, respectively [11]) can be reached using standard superconducting magnets, allowing one to experimentally investigate the phase diagram in detail [11-13]. The observation of a number of subsequent low-temperature fieldinduced transitions has triggered intensive theoretical studies ([5] and references herein), revealing the important role of $\mathcal{H}_{\delta}$ [Eq. (1)]. Very recently, several new field-induced transitions were observed in $\mathrm{Cs}_{2} \mathrm{CuCl}_{4}$, emerging under applied hydrostatic pressure [14].

Inelastic neutron-scattering experiments on $\mathrm{Cs}_{2} \mathrm{CuCl}_{4}$ revealed the presence of a highly dispersive continuum of excited states $[12,15]$. These states were initially identified as $2 \mathrm{D}$ RVB states, as suggested to occur in the AF system of spins on a triangular layer lattice [7]. However, later on, the data were reinterpreted in the framework of the quasi-1D Tomonaga-Luttinger spin-liquid scenario [16], with spinons (and their interchain bound-state excitations, triplons) as elementary magnetic excitations. A key condition here is the presence of the geometrical frustration, making spin chains 


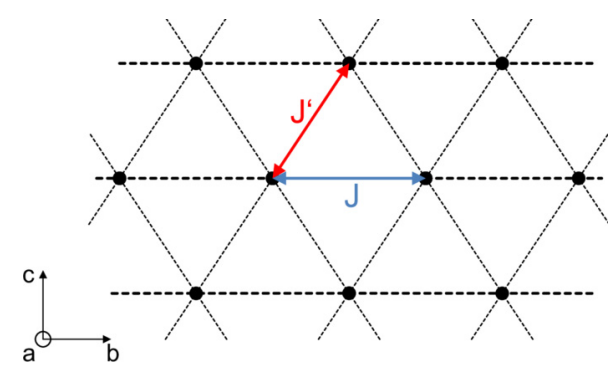

FIG. 1. Schematic view of exchange paths in $\mathrm{Cs}_{2} \mathrm{CuCl}_{4}$ with the exchange coupling $J$ along the $b$ axis (chain direction) and $J^{\prime}$ along the zigzag bonds in the $b c$ plane.

well isolated from each other (this is in contrast to the 2D Majorana spin-liquid scenario [17], with magnetic excitations, however, coherently propagating along the direction of the strongest exchange interaction). The quasi-1D TomonagaLuttinger spin-liquid scenario perfectly describes the overall picture of magnetic excitations in $\mathrm{Cs}_{2} \mathrm{CuCl}_{4}$, including their behavior in magnetic fields [18]. ESR studies have supported the proposed model, with the uniform DM interaction opening an energy gap $(\sim 0.7 \mathrm{~K})$ at the $\Gamma$ point in the quantumdisordered state [19].

Here, we report on heat-transport studies of $\mathrm{Cs}_{2} \mathrm{CuCl}_{4}$, with the main goal to probe the anisotropy of the thermal conductivity on the temperature scale $T \sim J / k_{B}$, where effects of the dimensionality of magnetic correlations should become relevant ([20] and references therein).

Single crystals of $\mathrm{Cs}_{2} \mathrm{CuCl}_{4}$ were grown by the slow evaporation of aqueous solution of $\mathrm{CsCl}$ and $\mathrm{CuCl}_{2}$ in the mole ratio $2: 1$. The room-temperature crystallographic parameters $(a=9.769 \AA, b=7.607 \AA$, and $c=12.381 \AA$; space group Pnma) were confirmed by x-ray diffraction and are in good agreement with those reported previously [21].

Thermal-conductivity measurements were performed in a ${ }^{3} \mathrm{He}$ cryostat, in magnetic fields up to $14 \mathrm{~T}$. Samples were prepared in a rodlike shape (with a thickness of about $0.5 \mathrm{~mm}$ and a length of about $5 \mathrm{~mm}$ ) to allow for a sufficiently high temperature gradient. The temperature difference was produced by a heater attached at one end of the sample and measured by a pair of matched $\mathrm{RuO}_{2}$ thermometers (as thermometers we used standard thick-film $\mathrm{RuO}_{2}$-based surface mount device resistors); a four-point technique was employed to measure the temperature gradient along the sample. To reduce the statistical error, one point in the thermal conductivity (at fixed temperature and magnetic field) was averaged over 20 measurement cycles. In the experiments, the magnetic field was applied along the direction of the heat transport. The temperature in the entire field range was measured with accuracy better than $\pm 5 \%$.

The thermal-conductivity experiments on $\mathrm{Cs}_{2} \mathrm{CuCl}_{4}$ revealed a broad maximum at about $5 \mathrm{~K}$ for all three directions (Fig. 2). Such a behavior is a textbook example of the phonon-dominated thermal conductivity, where the lowtemperature conductivity is determined by phonon scattering on the crystal boundaries and structural imperfections, while the high-temperature mean-free path is limited by the umklapp scattering [22]. As one can see, absolute values of the thermal conductivity (in particular at the maximum positions)
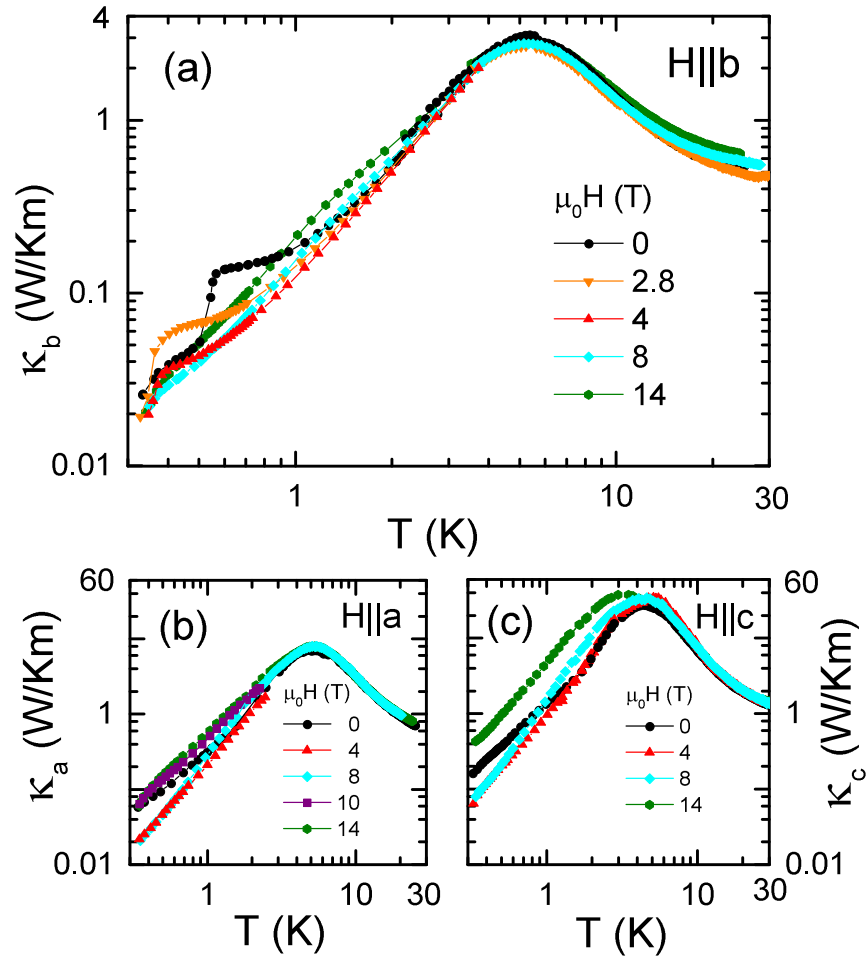

FIG. 2. Temperature dependence of the thermal conductivity $\kappa_{b}$ (a), $\kappa_{a}$ (b), and $\kappa_{c}$ (c) along the $b, a$, and $c$ axes, respectively, in selected magnetic fields. Lines are guides to the eye.

are different for different directions: the value is maximal for the direction along the $c$ axis and a few times smaller for the $a$ and $b$ directions. This difference can be tentatively explained by the anisotropy of sound velocity and phononmagnon scattering in $\mathrm{Cs}_{2} \mathrm{CuCl}_{4}$ at low temperatures.

With decreasing temperature we observed that the heattransport behavior along the $b$ direction becomes significantly different from that along the $a$ and $c$ directions. Along the $b$ axis, before entering the 3D-ordered phase, the thermal conductivity changes the slope (reflecting its pronounced enhancement, in contrast to that for other directions) and suddenly drops, once the material undergoes the $3 \mathrm{D}$ ordering. The anomaly position depends on the applied magnetic field, shifting to lower temperatures with increasing field (which is consistent with the temperature-field phase diagram obtained by Tokiwa et al. [11]). Based on these observations, we suggest that the anomaly of the thermal conductivity, revealed by us in the vicinity of $T_{N}$ specifically along the $b$ direction, has a magnetic nature and is determined by magnetic excitations propagating along the direction of the largest exchange interaction. For the Tomonaga-Luttinger spin liquid, proposed for $\mathrm{Cs}_{2} \mathrm{CuCl}_{4}$ [16], these excitations are spinons. Similar anisotropic behavior of the thermal transport was observed in quasi-1D chain materials $\mathrm{Sr}_{2} \mathrm{CuO}_{3}$ [23,24], $\mathrm{SrCuO}_{2}$ [24], $\mathrm{Cu}\left(\mathrm{C}_{4} \mathrm{H}_{4} \mathrm{~N}_{2}\right)\left(\mathrm{NO}_{3}\right)_{2}$ [25], and $\mathrm{CaCu}_{2} \mathrm{O}_{3}$ [26]. Noticeably, apart from the shift, the applied magnetic field suppresses the anomaly, making it almost undetectable at $8 \mathrm{~T}$. Such a behavior is consistent with the field-induced crossover from the quantum to a classically favored state, where quantum fluctuations are significantly suppressed by magnetic field [27]. 

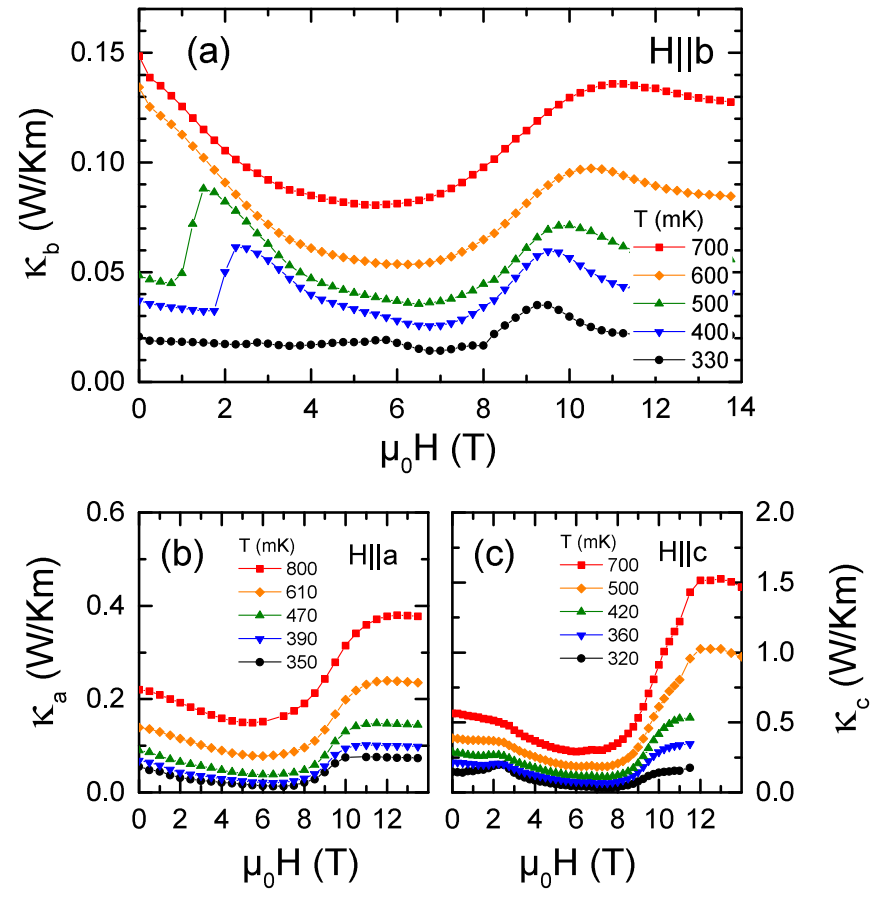

FIG. 3. Field dependence of the thermal conductivity $\kappa_{b}(\mathrm{a}), \kappa_{a}$ (b), and $\kappa_{c}$ (c) at selected temperatures. The magnetic field was applied along the heat-transport directions. Lines are guides to the eye.

The sudden drop of the thermal conductivity $\kappa_{b}$ at $T_{N}$ can be explained by a collapse of the $1 \mathrm{D}$ spinon-heat transport when entering the 3D AF-ordered state, accompanied by the opening of an energy gap in the low-temperature excitation spectrum. Such a gap $(\sim 1.3 \mathrm{~K})$ was observed in $\mathrm{Cs}_{2} \mathrm{CuCl}_{4}$ by means of ESR [28].

Field measurements revealed a nonmonotonic behavior of the thermal conductivity for all three directions of the applied magnetic fields (Fig. 3). For the heat transport along the $b$ and $c$ axes, we observe changes in the heat transport behavior, which occur in magnetic fields about $2 \mathrm{~T}$. These anomalies correspond to field-induced transitions from the 3D ordered to a disordered state, as reported previously [11-13]. Noticeable low-field jumps of $\kappa_{b}$ at about 0.5 and $0.4 \mathrm{~K}$ [Fig. 3(a)] provide additional evidence of the 1D nature of magnetic excitations in this material, significantly contributing to the heat transport just above $T_{N}$.

Another peculiar finding is the pronounced dip in the field dependencies of thermal conductivity at about 6-7 T, observed for all three directions (Fig. 3). This observation is consistent with the low-temperature ultrasound properties of $\mathrm{Cs}_{2} \mathrm{CuCl}_{4}$ [29-31], suggesting the exchange-striction mechanism as a possible reason of such a behavior. A pronounced increase of the thermal conductivity above $H_{\text {sat }}$ can be understood taking into account the decreased phonon-magnon scattering in the fully spin-polarized phase (the positions of corresponding maxima in thermal conductivity along the $b$ direction are shown in Fig. 4 by crosses). The phase diagram obtained by us, together with results of previously reported specific-heat and magnetic-susceptibility studies [11] for $H \| b$, is shown in Fig. 4; excellent agreement between the two data sets is found.

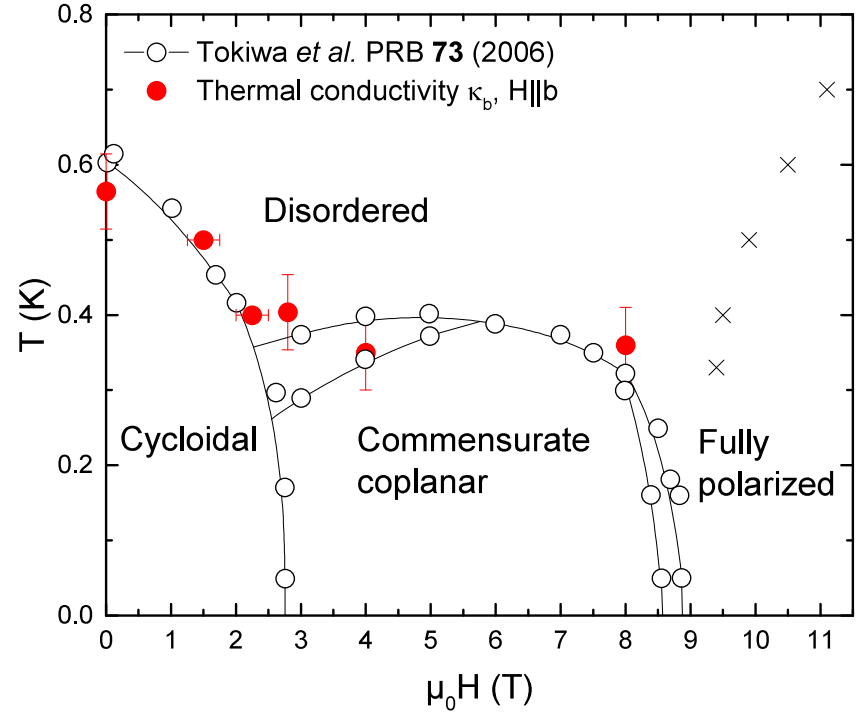

FIG. 4. Phase diagram of $\mathrm{Cs}_{2} \mathrm{CuCl}_{4}$ for magnetic field applied along the $b$ direction. Data from our thermal-transport measurements are shown by red solid circles, while specific-heat and susceptibility data from [11] are denoted by open circles. The crosses correspond to the thermal conductivity maxima in the fully spin-polarized phase above $H_{\text {sat }}$ (Fig. 3). Lines are guides to the eye.

To estimate the magnetic mean-free path $l_{b}^{m}$ along the $b$ direction, low-temperature heat transport properties of $\mathrm{Cs}_{2} \mathrm{CuCl}_{4}$ were analyzed in the framework of the spin-1/2 AF Heisenberg chain model [26] with spinons as elementary magnetic excitations:

$$
l_{b}^{m}=\left(\frac{\kappa_{b}^{m}}{T}\right)\left(\frac{3 \hbar}{N_{s} k_{B}^{2} \pi}\right) .
$$

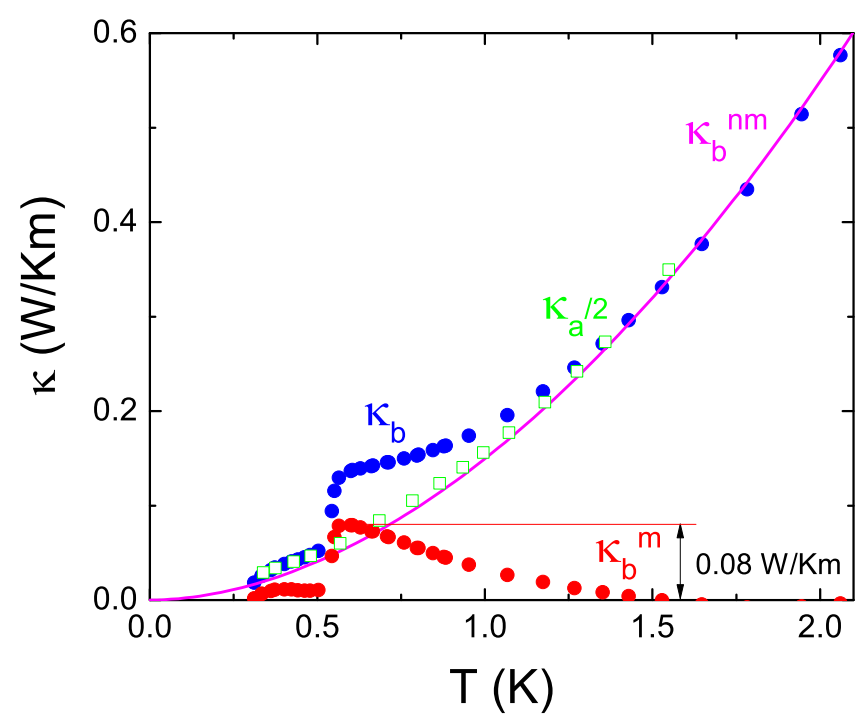

FIG. 5. Temperature dependencies of the thermal conductivity $\kappa_{b}$ along the $b$ axis (rough experimental data; blue symbols), nonmagnetic background $\kappa_{b}^{n m}$ as the result of the power-law fit (magenta line), magnetic contribution to the thermal conductivity $\kappa_{b}^{m}$ as the result of the subtraction $\kappa_{b}^{n m}$ from $\kappa_{b}$ (red symbols), and rescaled thermal conductivity $\kappa_{a} / 2$ along the $a$ direction (green symbols). 
Here, $\kappa_{b}^{m}$ is the magnetic heat conductivity, $\hbar$ is the reduced Planck constant, and $N_{s}=4 / a c$ is the number of spins per unit area. To estimate the magnetic heat conductivity $\kappa_{b}^{m}$ the nonmagnetic background $\kappa_{b}^{n m}$ was subtracted from the experimental data $\kappa_{b}$. To obtain $\kappa_{b}^{n m}$, the zero-field thermalconductivity data $\kappa_{b}$ below and above the anomaly were fitted with the power law $\kappa=0.1496 T^{1.875}$ (line $\kappa_{b}^{n m}$ in Fig. 5). For comparison, the rescaled thermal conductivity along the $a$ direction $\kappa_{a} / 2$ (where no anomaly was observed) is shown, revealing excellent agreement with the fit results. The subtraction results yield $0.08 \mathrm{~W} / \mathrm{K} \mathrm{m}$ for the magnetic part of the heat conductivity at the maximum position. Based on this model,we obtained $l_{m} \approx 200 \AA$, which corresponds to approximately 30 lattice spacings along the $b$ direction.

In conclusion, the thermal conductivity in $\mathrm{Cs}_{2} \mathrm{CuCl}_{4}$ was measured at temperatures down to $300 \mathrm{mK}$ in magnetic fields up to $14 \mathrm{~T}$ along the three principal crystallographic directions. The heat transport is found to be dominated by the phonon contribution. On the other hand, a pronounced field-dependent anomaly of the thermal conductivity was observed along the $b$ axis, when approaching the transition into the 3D-ordered state. The anomaly is attributed to the 1D heat transport through magnetic excitations propagating in $\mathrm{Cs}_{2} \mathrm{CuCl}_{4}$ along the direction of the strongest exchange coupling. Our observations strongly support the quasi-1D spin-liquid scenario with spinons as elementary excitations, proposed for this frustrated antiferromagnet.

This work was supported by the Deutsche Forschungsgemeinschaft (DFG), through ZV 6/2-2, the excellence cluster ct.qmat (EXC2147, Project ID 39085490), and SFB 1143, as well as by the HLD at HZDR, member of the European Magnetic Field Laboratory (EMFL). We acknowledge fruitful discussions with O. Starykh, T. Lorenz, X. Zotos, A. Chernyshev, A. Zheludev, S. Zherlitsyn, U. H. Acosta, and A. Pidatella.
[1] A. P. Ramirez, Annu. Rev. Mater. Sci. 24, 453 (1994).

[2] L. Balents, Nature (London) 464, 199 (2010).

[3] C. Lacroix, P. Mendels, and F. Mila, Introduction to Frustrated Magnetism (Springer-Verlag, Berlin, 2011).

[4] A. A. Zvyagin, Low Temp. Phys. 39, 901 (2013).

[5] O. A. Starykh, Rep. Prog. Phys. 78, 052502 (2015).

[6] J. Wosnitza, S. A. Zvyagin, and S. Zherlitsyn, Rep. Prog. Phys. 79, 074504 (2016).

[7] P. W. Anderson, Mater. Res. Bull. 8, 153 (1973).

[8] S. A. Zvyagin, D. Kamenskyi, M. Ozerov, J. Wosnitza, M. Ikeda, T. Fujita, M. Hagiwara, A. I. Smirnov, T. A. Soldatov, A. Y. Shapiro, J. Krzystek, R. Hu, H. Ryu, C. Petrovic, and M. E. Zhitomirsky, Phys. Rev. Lett. 112, 077206 (2014).

[9] R. Coldea, D. A. Tennant, K. Habicht, P. Smeibidl, C. Wolters, and Z. Tylczynski, Phys. Rev. Lett. 88, 137203 (2002).

[10] R. Coldea, D. A. Tennant, R. A. Cowley, D. F. McMorrow, B. Dorner, and Z. Tylczynski, J. Phys.: Condens. Matter 8, 7473 (1996).

[11] Y. Tokiwa, T. Radu, R. Coldea, H. Wilhelm, Z. Tylczynski, and F. Steglich, Phys. Rev. B 73, 134414 (2006).

[12] R. Coldea, D. A. Tennant, A. M. Tsvelik, and Z. Tylczynski, Phys. Rev. Lett. 86, 1335 (2001).

[13] S. T. Hannahs, N. A. Fortune, J.-H. Parks, Y. Takano, T. Ono, and H. Tanaka, J. Phys.: Conf. Ser. 568, 042010 (2014).

[14] S. A. Zvyagin, D. Graf, T. Sakurai, S. Kimura, H. Nojiri, J. Wosnitza, H. Ohta, T. Ono, and H. Tanaka, Nat. Commun. 10, 1064 (2019).

[15] R. Coldea, D. A. Tennant, and Z. Tylczynski, Phys. Rev. B 68, 134424 (2003).

[16] M. Kohno, O. A. Starykh, and L. Balents, Nat. Phys. 3, 790 (2007).
[17] T. Herfurth, S. Streib, and P. Kopietz, Phys. Rev. B 88, 174404 (2013).

[18] M. Kohno, Phys. Rev. Lett. 103, 197203 (2009).

[19] K. Yu. Povarov, A. I. Smirnov, O. A. Starykh, S. V. Petrov, and A. Ya. Shapiro, Phys. Rev. Lett. 107, 037204 (2011).

[20] C. Hess, Phys. Rep. 811, 1 (2019).

[21] S. Bailleul, D. Svoronos, P. Porchner, and A. Tomas, C. R. Acad. Sci. Ser. Gen., Ser. 2 313, 1149 (1991).

[22] C. Kittel, Introduction to Solid State Physics (Wiley, New York, 1956).

[23] A. V. Sologubenko, E. Felder, K. Giannò, H. R. Ott, A. Vietkine, and A. Revcolevschi, Phys. Rev. B 62, R6108 (2000).

[24] A. V. Sologubenko, K. Giannò, H. R. Ott, A. Vietkine, and A. Revcolevschi, Phys. Rev. B 64, 054412 (2001).

[25] A. V. Sologubenko, K. Berggold, T. Lorenz, A. Rosch, E. Shimshoni, M. D. Phillips, and M. M. Turnbull, Phys. Rev. Lett. 98, 107201 (2007)

[26] C. Hess, H. ElHaes, A. Waske, B. Büchner, C. Sekar, G. Krabbes, F. Heidrich-Meisner, and W. Brenig, Phys. Rev. Lett. 98, 027201 (2007).

[27] O. A. Starykh, W. Jin, and A. V. Chubukov, Phys. Rev. Lett. 113, 087204 (2014).

[28] A. I. Smirnov, K. Yu. Povarov, S. V. Petrov, and A. Ya. Shapiro, Phys. Rev. B 85, 184423 (2012).

[29] A. Sytcheva, O. Chiatti, J. Wosnitza, S. Zherlitsyn, A. A. Zvyagin, R. Coldea, and Z. Tylczynski, Phys. Rev. B 80 , 224414 (2009).

[30] S. Streib, P. Kopietz, P. T. Cong, B. Wolf, M. Lang, N. van Well, F. Ritter, and W. Assmus, Phys. Rev. B 91, 041108(R) (2015).

[31] O. Tsyplyatyev, P. Kopietz, Y. Tsui, B. Wolf, P. T. Cong, N. van Well, F. Ritter, C. Krellner, W. Assmus, and M. Lang, Phys. Rev. B 95, 045120 (2017). 